\title{
AUXILIARY LIVER TRANSPLANT IN A PEDIATRIC PATIENT FOR ACUTE LIVER FAILURE SECONDARY TO TUBERCULOSIS TREATMENT
}

\section{Euroanaesthesid 18}

Department of Anesthesia and Critical Care, Clínica Universidad de Navarra. Pamplona, Spain.

\section{Introduction}

The potential risk of hepatotoxicity of Isoniazid (INH), Rifampicin (RMP) and Pyrazinamide (PZA) in the first-line treatment of tuberculosis has been described extensively. Despite of this, fulminant liver failure (FLF) due to toxic causes is an exceptional entity in paediatrics, with a rapidly progressive course and poor prognosis. The only therapy in most cases is transplantation, a treatment considered to be the gold standard, increasing survival to more than $60 \%$ in one year. We present a case of FHF due to antituberculous treatment in a 12-year-old boy, who required auxiliary orthotopic liver transplant (AOLT).

\section{Case description}

A 12-year-old male from Bolivia, with no relevant past medical history, presented with a 2 weeks of productive morning cough associated with fever, night sweats and poor general condition. Chest x-rays showed a cavitated multilobar pneumonia, suggestive of tuberculosis. After initiating antituberculous treatment, he presented FLF with coagulopathy and severe encephalopathy. An organ for transplant was urgently requested
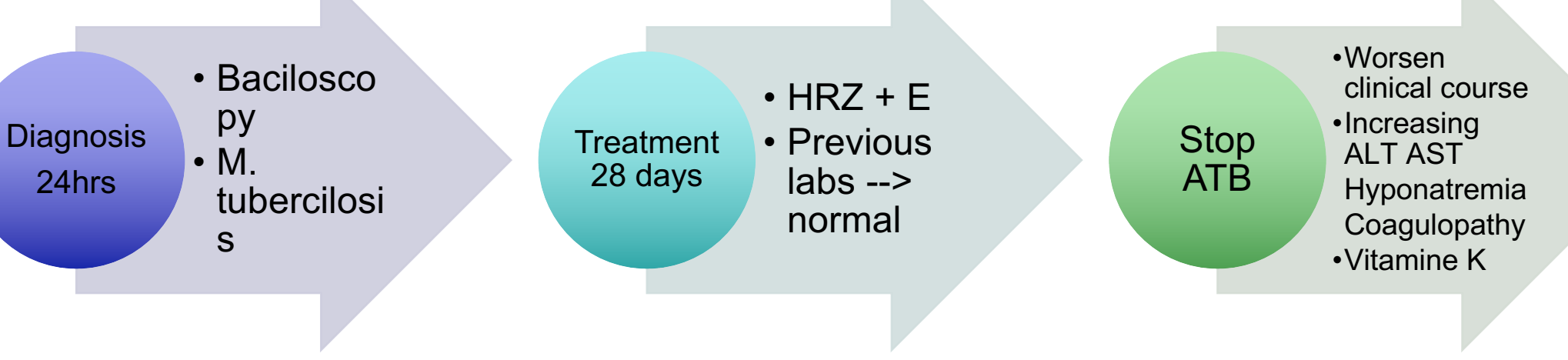

\section{Surgical technique}

-A whole or reduced organ was used, by implanting left liver, eliminating segments II. III and IV.

-The graft was implanted in the right upper quadrant, with the hilum located medially and the posterior vena cava.

-The inferior vena cava of the donor was attached end-to-side with the receptor's cava, in the highest possible situation.

\section{Anesthetic management}

-Balanced General anesthesia. Intubated previously in the ICU.

-Invasive monitorization (including Swan Ganz catheter)

-Politransfusion + Prothrombin complex concentrate 3000ui.

-ATB prophylaxis : Vancomicine + Metronidazole + Anidulafungin

-Inotropes: Dobutamine.

\section{Evolution}

After surgery, the child presented progressive improvement of general condition despite neurological sequelae with $6^{\circ}$ pair palsy and right hemiparesis that resolved.

-Liver function tests showed decreased ALT/AST and bilurubin, associated with increased hepatic synthesis (graphs).

-Liver biopsy 15 days post-transplant, reports viability of native liver without signs of regeneration and acute rejection of the donor organ, which was treated with corticosteroids.

\section{Conclusions}

AOLT is a safe therapeutic alternative in paediatric patients with active tuberculosis and FLF secondary to antituberculous treatment. The start of Immunosuppression and the reintroduction of tuberculostatic drugs are a challenge to avoid new complications.

R. Schneider, S. Bercker, S. Schubert H. L. Tillmann, J 